\title{
Thermal transport in sheared electro- and magnetorheological fluids
}

\author{
Martin C. Heine \\ Particle Technology Laboratory, Department of Mechanical and Process Engineering, ETH Zürich, \\ 8092 Zürich, Switzerland \\ Juan de Vicente \\ Department of Applied Physics, Faculty of Sciences, University of Granada, C/Fuentenueva s/n, \\ 18071-Granada, Spain \\ D. J. Klingenberg \\ Department of Chemical and Biological Engineering and Rheology Research Center, \\ University of Wisconsin, 1415 Engineering Drive, Madison, Wisconsin 53706
}

(Received 24 June 2005; accepted 9 January 2006; published online 17 February 2006)

\begin{abstract}
Thermal energy transport in sheared electrorheological and magnetorheological (ER and MR) fluids is analyzed. Although energy production by viscous dissipation can be significant, energy transport on the particle length scale can be analyzed by ignoring viscous dissipation. For typical situations, energy transport normal to the flow direction is dominated by conduction. Particle-level simulations were employed to determine the suspension structure as a function of Mason number and volume fraction. A self-consistent mean-field dipole model is used to estimate the effective thermal conductivities for these simulated structures. The field-induced chain-like aggregates that form at small Mason number result in a larger effective thermal conductivity at small Mason number than at large Mason number. Effects of higher-order multipoles are estimated by analyzing effective thermal conductivities of model structures. For highly conducting particles, the effective thermal conductivity of a sheared ER or MR suspension is predicted to roughly double as the Mason number is decreased from the large to the small Mason number limits. (C) 2006 American Institute of Physics. [DOI: 10.1063/1.2171442]
\end{abstract}

\section{INTRODUCTION}

Electro- and magnetorheological suspensions exhibit significant increases in apparent shear viscosity when exposed to external electric or magnetic fields, respectively, applied transverse to the direction of flow. This phenomenon has been exploited in controllable shock absorbers, and numerous other damping and torque transfer applications have been proposed. ${ }^{1-4}$

The large field-induced increase in apparent viscosity can give rise to significant energy production by viscous dissipation. Field-induced yield stresses as large as $10^{5} \mathrm{~Pa}$ have been reported for MR fluids. ${ }^{5,6}$ For a shear rate of $10^{3} \mathrm{~s}^{-1}$, this corresponds to energy production rates on the order of $S_{v} \sim 10^{8} \mathrm{~W} / \mathrm{m}^{3}$. Such rates are more than sufficient to produce significant temperature increases within the fluids and devices, particularly for devices in which the fluid is sheared between concentric cylinders. In this geometry, fluid elements remain in the shear flow for extended periods, allowing time for the fluid temperature to increase. In contrast, fluid elements in pressure-driven flow devices (e.g., shock absorbers) remain in the shearing flow for only a short period, and thus fluid temperature increases may not be as large.

The behavior of ER and MR fluids depends upon the temperature. For both types of fluids, the viscosity in the absence of an applied field varies with the temperature. For ER fluids, the electrical conductivity typically increases with temperature. Although the magnetic and field-induced rheo- logical properties of MR fluids are largely insensitive to variations in temperature of short duration, the rate of oxidation of iron increases with temperature. It is thus apparent that designing ER and MR devices to limit operating temperatures can have substantial benefits.

Typical ER and MR devices are composed of metal, because of the need to apply large electric and magnetic fields. Thus the fluid itself constitutes a significant resistance to the transport of thermal energy to the surroundings. Understanding energy transport in ER and MR fluids is thus important for designing MR devices, and avoiding deleterious effects associated with large temperatures. However, little is known about energy transport within these materials.

A considerable amount is known about energy transport in quiescent suspensions through experimental and theoretical investigations, of energy transport as well as the analogous problems of electrical and magnetic transport in suspensions. Consider spheres of radius $a$, volume fraction $\phi$, and thermal conductivity $k_{p}$, suspended randomly in a continuous phase of thermal conductivity $k_{c}$. The apparent, or effective, thermal conductivity of the suspension is often represented by Maxwell's approximate formula ${ }^{7,8}$

$$
\frac{k_{\mathrm{eff}}^{\mathrm{Max}}}{k_{c}}=\frac{1+2 \beta \phi}{1-\beta \phi},
$$

where $\beta=(\alpha-1) /(\alpha+2)$, and $\alpha=k_{p} / k_{c}$. This expression agrees well with experimental data for $\alpha \lesssim 10$ for all $\phi .{ }^{8,9}$ 
For $\alpha \gg 1$, the agreement is good for $\phi \leqq 0.2$. Chiew and Glandt ${ }^{8}$ derived the expression

$$
\frac{k_{\mathrm{eff}}^{\mathrm{CG}}}{k_{c}}=\frac{1+2 \beta \phi+\left(K_{2}-3 \beta^{2}\right) \phi^{2}}{1-\beta \phi},
$$

where $K_{2}$ is a known function of $\alpha$ and $\phi$. This expression agrees much better with experimental data for $\alpha \gg 1$ for all volume fractions.

Zuzovksy and Brenner ${ }^{10}$ analyzed the effective conductivity of suspensions of spheres on a cubic lattice. They obtained the formula

$$
\begin{aligned}
\frac{k_{\mathrm{eff}}^{\mathrm{ZB}}}{k_{c}}= & 1+3 \beta \phi\left[1-\beta \phi+\frac{1.3060 \beta \phi^{10 / 3}}{\frac{4 / 3+\alpha}{1-\alpha}+0.4072 \phi^{7 / 3}}\right. \\
& \left.+\frac{0.02218 \beta(1-\alpha) \phi^{14 / 3}}{6 / 5+\alpha}+\mathcal{O}\left(\phi^{6}\right)\right]^{-1} .
\end{aligned}
$$

This expression agrees with the experimental data reported by Meredith and Tobias ${ }^{11}$ for $\alpha \rightarrow 0$ for all $\phi$, and for $\alpha$ $\rightarrow \infty$ for $\phi \leqq 0.45$.

In shear flows, energy transport in directions orthogonal to the flow direction can be altered, even when the macroscopic flow is laminar. Ahuja ${ }^{12,13}$ investigated the effective thermal conductivity of suspensions in laminar, pressuredriven flow in tubes with circular cross section. For sufficiently small particle diameters and flow rates, the effective thermal conductivity in the radial direction was independent of flow rate. For larger particles and flow rates, the effective thermal conductivities increased. Similar observations were reported by Shin and $\mathrm{Lee}^{14}$ for suspensions in laminar circular Couette flow. For sufficiently small particle diameters and shear rates, the effective thermal conductivity in the radial direction was constant, but increased for larger particle diameters and shear rates. Zydney and Colton ${ }^{15}$ analyzed the augmentation of energy and mass transport in sheared suspensions. Mechanisms for enhanced transport in laminar flows include the convection caused by the particle rotation, and the shear-induced migration of particles and fluid elements. The augmentation of the thermal or mass diffusivity is predicted to scale with $a^{2} \dot{\gamma}$, which is qualitatively consistent with the experimental data reported by Ahuja and Shin and Lee; measurable enhancement in the effective thermal conductivity should only appear for sufficiently large $a$ and $\dot{\gamma}$.

Application of external electric or magnetic fields to quiescent ER and MR fluids, respectively, causes the formation of columnar aggregates oriented parallel to the applied field. Transport in this direction is thus altered. While no experimental information about the thermal conductivity is available, several experimental studies have reported enhancement of the electrical conductivity, ${ }^{16-18}$ permittivity, ${ }^{18,19}$ and magnetic permeability ${ }^{20,21}$ in the field direction relative to the respective properties in the absence of an applied field. Theoretical predictions of the increased effective transport properties of suspensions of chain-like structures agree qualitatively, ${ }^{22-24}$ predicting an enhancement that increases with increasing $\alpha$.

Several investigations of the effect of combined shear and electric fields on electrical transport in ER fluids have been reported. ${ }^{17,25-28}$ All studies report that the effective transport properties are enhanced at small shear rates and large field strengths, much like that observed for quiescent suspensions in applied fields. As the shear rate is increased, the effective transport properties decrease, approaching the values obtained for suspensions in the absence of a field. To the best of our knowledge, no studies of the combined effects of shear and electric or magnetic fields on the thermal transport properties have been reported.

In this article, we examine energy transport in suspensions exposed simultaneously to shear flow and external electric or magnetic fields. In the following section, we show that for typical ER and MR fluid applications, energy transport in the field direction is dominated by conduction. Furthermore, on the particle length scale, energy production by viscous dissipation can be ignored. Thus energy transport in the field direction can be described entirely by the effective thermal conductivity, which depends on the applied field strength and shear rate. Particle-level simulations are employed to determine the suspension structure, from which the effective thermal conductivity can be estimated using a selfconsistent mean-field dipole model. Results are consistent with experimental results for electrical transport. For small shear rates and large field strengths, the effective conductivity is larger than that of a quiescent suspension in the absence of a field. The effective conductivity decreases as the shear rate is increased or the field strength is decreased. The dependence on both shear rate and field strength can be expressed in terms of the dependence on the Mason number, which for ER fluids can be written $\mathrm{Mn}=\eta_{c} \dot{\gamma} /\left(2 \epsilon_{0} \epsilon_{c} \beta_{E}^{2} E_{0}^{2}\right)$ (see the following section for variable definitions). Corrections to the results from the dipole model are obtained using more accurate solutions of the temperature distribution and effective conductivity of idealized structures. For large $\alpha$, the effective thermal conductivity of a suspension at small $\mathrm{Mn}$ is predicted to be roughly twice as large as the effective thermal conductivity at large Mn (or quiescent, nonelectrified suspensions).

\section{ANALYSIS}

Consider a suspension of neutrally buoyant, monodisperse spheres in a Newtonian continuous phase. Assuming that the physical properties of each phase are independent of temperature, the thermal energy balance for each phase may be written ${ }^{29}$

$$
\rho \hat{C}_{p}\left(\frac{\partial T}{\partial t}+\boldsymbol{u} \cdot \nabla T\right)=k \nabla^{2} T+S_{v},
$$

where $\rho$ is the density, $\hat{C}_{p}$ is the specific constant pressure heat capacity, $T$ is the temperature, $\boldsymbol{u}$ is the velocity, $k$ is the thermal conductivity, and $S_{v}$ is rate of thermal energy production by viscous dissipation per unit volume $(=0$ within the particulate phase). 
TABLE I. Typical values for material properties for MR and ER fluids. $\mathrm{Pe}=a^{2} \dot{\gamma} / \alpha_{t}$ calculated for $a=1$ $\times 10^{-6} \mathrm{~m}$, and $\dot{\gamma}=10^{3} \mathrm{~s}^{-1}$.

\begin{tabular}{lccccc}
\hline \hline Material & $k(\mathrm{~W} / \mathrm{mK})$ & $\rho\left(\mathrm{kg} / \mathrm{m}^{3}\right)$ & $\hat{C}_{p}(\mathrm{~J} / \mathrm{kg} \mathrm{K})$ & $\alpha_{t}\left(\mathrm{~m}^{2} / \mathrm{s}\right)$ & $\mathrm{Pe}$ \\
\hline Iron $^{\mathrm{a}}$ & 80.2 & 7870 & 447 & $2.28 \times 10^{-5}$ & $4.39 \times 10^{-5}$ \\
Silica $^{\mathrm{b}}$ & 1.38 & 2220 & 745 & $8.34 \times 10^{-7}$ & $1.20 \times 10^{-3}$ \\
Titania $^{\mathrm{c}}$ & 8.4 & 4150 & 700 & $2.89 \times 10^{-6}$ & $3.46 \times 10^{-4}$ \\
Polystyrene $^{\mathrm{d}}$ & 0.14 & 1000 & 1300 & $1.08 \times 10^{-7}$ & $9.29 \times 10^{-3}$ \\
Engine oil $^{\mathrm{e}}$ & 0.145 & 884 & 1909 & $8.59 \times 10^{-8}$ & $1.16 \times 10^{-2}$ \\
Decane $^{\mathrm{f}}$ & 0.140 & 730 & 2211 & $8.67 \times 10^{-8}$ & $1.15 \times 10^{-2}$ \\
\hline \hline
\end{tabular}

a00 K, Ref. 30.

${ }^{\mathrm{b}}$ Fused, polycrystalline, $300 \mathrm{~K}$, Ref. 30.

'303 K, Ref. 31.

d 303 K, Ref. 31.

e 300 K, Ref. 30.

f $293-303$ K, Ref. 32.

Scaling length and time with the particle radius $a$ and the inverse shear rate $\dot{\gamma}^{-1}$, respectively, the energy balance can be written in dimensionless form as (dimensionless quantities are represented with a tilde)

$$
\operatorname{Pe}\left(\frac{\partial \theta}{\partial \widetilde{t}}+\widetilde{\mathbf{u}} \cdot \widetilde{\nabla} \theta\right)=\widetilde{\nabla}^{2} \theta+\widetilde{S}_{v},
$$

where $\mathrm{Pe}=a^{2} \dot{\gamma} / \alpha_{t}$ is the Peclet number for energy transport, $\alpha_{t}=k / \rho \hat{C}_{p}$ is the thermal diffusivity,

$$
\theta \equiv \frac{T-T_{R 1}}{T_{R 2}}
$$

is the dimensionless temperature, where $T_{R 1}$ is a reference temperature, and $T_{R 2}$ is characteristic temperature or temperature difference, and $\tilde{S}_{v}=a^{2} S_{v} /\left(k T_{R 2}\right)$.

Typical values for material properties of disperse and continuous phases commonly employed in ER and MR suspensions are listed in Table I. Also listed in Table I are representative values of the Peclet number for $a=1 \times 10^{-6} \mathrm{~m}$ and $\dot{\gamma}=10^{3} \mathrm{~s}^{-1}$. Even for such large shear rates, $\mathrm{Pe} \leq 10^{-2}$ for each phase. For such small Pe, the rate of energy transport by conduction on the length scale of the particle radius is much greater than the rate of energy transport by convection. It is thus apparent that the thermal transport characteristics of suspensions at small Peclet number will often be of importance. We will therefore examine the behavior for $\mathrm{Pe}=0$ from here forward. Neglecting the left-hand side of Eq. (5) yields

$$
\tilde{\nabla}^{2} \theta=-\tilde{S}_{v} .
$$

The important implication of the analysis thus far is that the temperature distribution is always essentially at steady state, even for flowing suspensions.

The rate of energy production by viscous dissipation may be estimated from macroscopic measurements of the shear stress $\tau$ and shear rate via $S_{v}=\tau \dot{\gamma}$. The largest shear stresses are obtained for concentrated MR suspensions for which yield stresses on the order of $10^{5} \mathrm{~Pa}$ have been reported. ${ }^{5,6}$ Under such conditions, the shear stress is dominated by the yield stress, and thus $\tau \approx 10^{5} \mathrm{~Pa}$ is a realistic upper limit for the shear stress magnitude. For shear rates of $\dot{\gamma}=10^{3}$, the rate of energy production by viscous dissipation is expected to be as large as $S_{v}=10^{8} \mathrm{~W} / \mathrm{m}^{3}$. The dimensionless rate of energy production within the continuous phase is thus $\tilde{S}_{v}=a^{2} S_{v} /\left(k T_{R 2}\right) \approx 7 \times 10^{-4}$, using $k=0.145 \mathrm{~W} / \mathrm{mK}$ which is appropriate for oil, and a characteristic temperature difference of $T_{R 2}=1 \mathrm{~K}$. We thus conclude that energy production by viscous dissipation does not significantly affect the temperature distribution on the particle length scale. Note that this does not contradict the statement in the Introduction that energy production by viscous dissipation can produce large increases in the suspension temperature. Rather, $\tilde{S}_{v}$ $\ll 1$ implies that energy conducts much more rapidly than it is produced on the particle scale. Thus while viscous dissipation influences the magnitude of the temperature, the locally averaged temperature profile remains spatially linear. This further implies that the impact of energy production by viscous dissipation may be determined by calculating the effective medium properties in the absence of energy production, and then solving for the effective medium temperature distribution throughout the entire suspension including energy production by viscous dissipation. This is illustrated in the Appendix, where the temperature distribution in a onedimensional heterogeneous mixture is determined exactly, and by employing the effective medium procedure outlined above. As long as $\widetilde{S}_{v} \ll 1$, the effective medium procedure is accurate, even when viscous dissipation significantly increases the suspension temperature.

Because $\widetilde{S}_{v}$ is small, the energy production term can be neglected, and the temperature distribution within the suspension is governed by

$$
\widetilde{\nabla}^{2} \theta=0
$$

even when the suspension is being sheared. Once Eq. (8) has solved, using appropriate boundary conditions, to obtain the temperature distribution throughout the suspension, the energy flux can be analyzed to obtain an effective thermal conductivity of the suspension.

We are primarily interested in energy flux in the direction of the applied electric of magnetic field, defined here as the $z$ direction (e.g., energy transport in the radial direction of a concentric cylinder device). The energy flux in the $z$ 
direction, $q_{z}$, in general can have contributions from conduction as well as convection,

$$
q_{z}=-k \frac{\partial T}{\partial z}+\rho \hat{C}_{p} u_{z}\left(T-T_{R 1}\right) .
$$

Nondimensionalizing as above, the flux can be rewritten

$$
q_{z}=\frac{k T_{R 2}}{a}\left[-\frac{\partial \theta}{\partial \widetilde{z}}+\operatorname{Pe} \widetilde{u}_{z} \theta\right] .
$$

As discussed above, Pe is typically small, and thus the energy flux in each phase will be dominated by conduction.

The average heat flux can be written as a volume average $^{7,33-35}$

$$
\begin{aligned}
\left\langle q_{z}\right\rangle & =-\frac{1}{V} \int_{V} k \frac{\partial T}{\partial z} d V \\
& =-k_{c}\left\langle\frac{\partial T}{\partial z}\right\rangle-n\left\langle S_{z}\right\rangle,
\end{aligned}
$$

where $V$ is the suspension volume, $n$ is the particle number density, $\langle\partial T / \partial z\rangle$ is the macroscopic temperature gradient, and

$$
\left\langle S_{z}\right\rangle \equiv \frac{1}{N} \sum_{i=1}^{N} \int_{V_{p}}\left(k_{p}-k_{c}\right) \frac{\partial T}{\partial z} d V
$$

is the "thermal dipole strength," where $V_{p}$ is the volume of a particle, and $N$ is the number of particles. The $z z$ component of the effective thermal conductivity, $k_{z z \text {,eff }} \equiv k_{\text {eff }}$, is then given by

$$
\begin{aligned}
k_{\mathrm{eff}} & =\frac{\left\langle q_{z}\right\rangle}{-\langle\partial T / \partial z\rangle} \\
& =k_{c}+\frac{n\left\langle S_{z}\right\rangle}{\langle\partial T / \partial z\rangle} .
\end{aligned}
$$

To calculate the effective thermal conductivity, one must therefore first solve for the temperature distribution throughout the suspension, or rather, the temperature gradient in each particle. In this article, this is done using two different methods. We employ particle-level simulations to first generate "snapshots" of the suspension microstructure, and then use a self-consistent, mean-field (SCMF) approximation to determine the temperature gradient in each particle. The effective thermal conductivity is then obtained using Eqs. (13) and (15). Similar calculations with idealized suspension structures composed of chains of particles on a lattice will also be performed to probe the effects of anisotropy on the thermal transport. For these idealized structures, we also evaluate the effective thermal conductivity using the finite element method, in order to assess the accuracy of the SCMF results. For these calculations, we use the software packages ANSYS and FEMLAB to determine the temperature distribution in a unit cell of the lattice, from which the effective thermal conductivity can be evaluated directly using Eq. (14). The meshes were reduced until the uncertainty in the calculated thermal conductivity was less than $1 \%$.
In the following section, we briefly describe the simulation method and the SCMF method for evaluating the effective thermal conductivity of the suspension. Results are presented in Sec. IV.

\section{METHODS}

\section{A. Simulation method}

We employ a particle-level simulation method described previously. ${ }^{36-38}$ The suspension is modeled as a system of $N$ non-Brownian, neutrally buoyant spheres (diameter $\sigma=2 a$ ) between bounding surfaces located at $z= \pm L_{z} / 2$, with the electric/magnetic field applied in the $z$ direction. The motion of each sphere is governed by Newton's Law of translational motion. Field-induced forces are approximated in the pointdipole limit, where the force on particle $i$ at the origin caused by particle $j$ at $r_{i j}, \theta_{i j}$ is given in the point-dipole limit by ${ }^{36,39}$

$$
\boldsymbol{F}_{i j}^{\mathrm{el} . / \mathrm{mag} .}=F_{0}\left(\frac{\sigma}{r_{i j}}\right)^{4}\left[\left(3 \cos ^{2} \theta_{i j}-1\right) \boldsymbol{e}_{r}+\sin 2 \theta_{i j} \boldsymbol{e}_{\theta}\right],
$$

where $\theta_{i j}$ is the angle between the line-of-centers and the applied electric field $\boldsymbol{E}_{0}$ or the magnetic field $\boldsymbol{H}_{0}$, and $\boldsymbol{e}_{r}$ and $\boldsymbol{e}_{\theta}$ are unit vectors in the direction of increasing $r_{i j}$ and $\theta_{i j}$, respectively.

Assuming linear polarization, the force magnitude $F_{0}$ for ER fluids is

$$
F_{0}=\frac{3}{16} \pi \epsilon_{0} \epsilon_{c} \sigma^{2} \beta_{E}^{2} E_{0}^{2},
$$

where $\epsilon_{0}=8.8542 \times 10^{-12} \mathrm{~F} / \mathrm{m}$ is the permittivity of free space, and $\epsilon_{c}$ is the relative dielectric constant of the continuous phase. The term $\beta_{E}$ is $\left(\sigma_{p}-\sigma_{c}\right) /\left(\sigma_{p}+2 \sigma_{c}\right)$ in dc fields, where $\sigma_{p}$ and $\sigma_{c}$ are the electrical conductivities of the disperse and continuous phases. In high frequency ac fields, $\beta$ $=\left(\epsilon_{p}-\epsilon_{c}\right) /\left(\epsilon_{p}+2 \epsilon_{c}\right)$, where $\epsilon_{p}$ is the relative dielectric constant of the particulate material [at large frequencies the force and field strength in Eqs. (16) and (17) represent the rms values]. For two spheres in a magnetic field, the pointdipole force magnitude assuming linear magnetization is ${ }^{40,41}$

$$
F_{0}=\frac{3}{16} \pi \mu_{0} \mu_{c} \sigma^{2} \beta_{M}^{2} H_{0}^{2},
$$

where $\mu_{0}=4 \pi \times 10^{-7} \mathrm{~N} / \mathrm{A}^{2}$ is the magnetic permeability of free space, $\mu_{c}$ is the relative permeability of the continuous phase, and $\beta_{M}=\left(\mu_{p}-\mu_{c}\right) /\left(\mu_{p}+2 \mu_{c}\right)$, where $\mu_{p}$ is the relative permeability of the particulate material.

The neglect of higher-order electric or magnetic multipoles in the point-dipole force expressions above underestimates the magnitude of the attractive force between particle pairs aligned with an applied field. ${ }^{42-44}$ However, even if these multipoles are taken into account, the scaling of the pair force with field strength and particle size are still given by the dipole results, e.g., $\left|\boldsymbol{F}^{\text {el. }}\right| \propto \sigma^{2} E_{0}^{2}$ for ER fluids. For interactions among many particles, many-body interactions also tend to result in pair forces larger than that obtained by a simple pairwise summation of the forces given by Eq. (16). ${ }^{42,43,45}$

For particles in magnetic fields, the point-dipole approximation above also neglects the nonlinear magnetization exhibited by typical ferromagnetic materials. ${ }^{2,46}$ However, at 
sufficiently large field strengths, the particle magnetization saturates. In this limit, the dipole force expression [Eq. (16)] becomes exact, with the force magnitude given by ${ }^{40,41}$

$$
F_{0}=\frac{1}{48} \pi \mu_{0} \mu_{c} \sigma^{2} M_{s}^{2},
$$

where $M_{s}$ is the saturation magnetization.

Short-range repulsive forces are given by the function $\boldsymbol{F}_{i j}^{\text {rep. }}=F_{0} \exp \left[-100\left(r_{i j} / \sigma-1\right)\right]\left(-\boldsymbol{e}_{r}\right)$, to mimic a hard-sphere repulsion. Similar short-range repulsive forces between each sphere and the bounding surfaces are imposed. The hydrodynamic force is given by Stokes drag, $\boldsymbol{F}_{i}^{\mathrm{hyd}}=-3 \pi \eta_{c} \sigma\left(\boldsymbol{u}_{i}\right.$ $\left.-\boldsymbol{u}^{\infty}\left(\boldsymbol{x}_{i}\right)\right)$, where the ambient flow is a simple shear with $\boldsymbol{u}^{\infty}(\boldsymbol{x})=\dot{\gamma}\left(z+L_{z} / 2\right) \boldsymbol{e}_{x}$, where $\dot{\gamma}$ is the shear rate.

The equation of motion of each sphere is integrated numerically via an explicit Euler method. Spheres with surfaces within a distance $0.05 \sigma$ of a bounding surface are assumed to be stuck, and assume the lateral velocity of the surface. Simulations reported here were performed for initially random suspensions contained between bounding surfaces in a simulation cell of dimensions $L_{x} \times L_{y} \times L_{z}=20 \sigma \times 10 \sigma$ $\times 10 \sigma$, periodic in the $x$ and $y$ directions.

The shear rate and field strength employed in the simulations can be characterized by a single dimensionless group known as the Mason number, $\mathrm{Mn}=3 \pi \eta_{c} \sigma^{2} \dot{\gamma} /\left(32 F_{0}\right)$. Using Eq. (17) for $F_{0}$ for ER fluids,

$$
\mathrm{Mn}=\frac{\eta_{c} \dot{\gamma}}{2 \epsilon_{0} \epsilon_{c} \beta_{E}^{2} E_{0}^{2}} .
$$

For MR fluids, the Mason number is Mn $=\eta_{c} \dot{\gamma} /\left(2 \mu_{0} \mu_{c} \beta_{M}^{2} H_{0}^{2}\right)$ or $9 \eta_{c} \dot{\gamma} /\left(2 \mu_{0} \mu_{c} M_{s}^{2}\right)$, for linear and saturated magnetization, respectively.

\section{B. Self-consistent mean-field analysis}

Consider first the temperature distribution caused by an isolated sphere of radius $a$ at the origin in an otherwise uniform ambient temperature gradient, $T^{\infty}(\boldsymbol{x})=\boldsymbol{G} \cdot \boldsymbol{x}$. Solution of Eq. (8) with the appropriate boundary conditions yields the temperature distribution ${ }^{7}$

$$
T(\boldsymbol{x})=\left\{\begin{array}{cc}
\left(1-\beta \frac{a^{3}}{|\boldsymbol{x}|^{3}}\right) \boldsymbol{G} \cdot \boldsymbol{x} & \text { for }|\boldsymbol{x}|>a, \\
(1-\beta) \boldsymbol{G} \cdot \boldsymbol{x} & \text { for }|\boldsymbol{x}| \leqslant a .
\end{array}\right.
$$

Equation (21) illustrates the temperature disturbance external to the sphere is that of a dipole temperature field emanating from the sphere center. Substituting Eq. (21) into Eq. (13) gives the dipole strength,

$$
S=4 \pi a^{3} k_{c} \beta \boldsymbol{G} .
$$

Assuming that a dilute suspension of spheres can be treated as a collection of isolated spheres, substitution of Eq. (22) in Eq. (15) gives the effective conductivity of a dilute suspension

$$
\lim _{\phi \rightarrow 0} \frac{k_{\mathrm{eff}}}{k_{c}}=1+3 \beta \phi
$$

For a nondilute suspension, we employ the SCMF approximation described by Jeffrey, ${ }^{7}$ and employed by Adriani and Gast ${ }^{45}$ in a model of the dielectric properties of Brownian ER suspensions. Assuming that the polarization of each sphere may be treated as a dipole, the dipole strength can be determined approximately by modifying Eq. (22). The dipole strength is written as the thermal polarizability $4 \pi a^{3} k_{c} \beta$ times the local temperature gradient, defined as the applied temperature gradient $\boldsymbol{G}$ plus the gradients of the dipole disturbance fields of all of the other spheres. The effective thermal conductivity ( $z z$ component) can then be written

$$
\begin{aligned}
\frac{k_{\mathrm{eff}}}{k_{c}}= & 1+3 \beta \phi\left\{1-\beta \phi-\frac{3 \beta \phi}{4 \pi} \int_{r>2 a} \frac{3 \cos ^{2} \theta-1}{r^{3}}\right. \\
& \times[g(\boldsymbol{r})-1] d \boldsymbol{r}\}^{-1},
\end{aligned}
$$

where $\theta$ is the angle between the applied field and the centerto-center separation of a sphere pair $\boldsymbol{r}$, and $g(\boldsymbol{r})$ is the pair distribution function. For a structureless suspension $[g(\boldsymbol{r})$ $=1$ for $r>2 a$ ], Eq. (24) reduces to Maxwell's formula ${ }^{7}$

$$
\frac{k_{\mathrm{eff}}}{k_{c}}=\frac{1+2 \beta \phi}{1-\beta \phi},
$$

which describes thermal transport in isotropic suspensions reasonably well as discussed in the Introduction.

For structured, anisotropic ER and MR suspensions exposed to external fields, the integral in Eq. (24) must be evaluated. We do this using configurations (i.e., particle positions) obtained from the simulations. The pair distribution function is expressed ${ }^{47}$

$$
n g(\boldsymbol{r})=\left\langle\sum_{j \neq i} \delta\left(\boldsymbol{r}-\boldsymbol{r}_{i j}\right)\right\rangle,
$$

where $n$ is the particle number density, the sum is over all particles and periodic images within a volume of $50 \sigma$ $\times 50 \sigma \times 100 \sigma$ (excluding particle $i$ ), and the angled brackets represent an average over all particles $i$. This results in an estimate of $k_{\text {eff }} / k_{c}$ for each configuration obtained from the simulation.

In addition to structures obtained from the simulations, we also perform the above analysis for manufactured structures composed of particles in chains aligned with the applied field, arranged on a square two-dimensional lattice in the plane perpendicular to the field (the "perfect chain structure"), and for cubic arrays of spheres. These results are compared with those obtained for simulated structures to assess the degree of anisotropy. These results are also compared with those obtained from finite-element solutions using identical, periodic structures, to assess the accuracy of the SCMF approximation. For the perfect chain structure, neighboring particle centers within a chain are separated by $1.03 \sigma$.

\section{RESULTS AND DISCUSSION}

\section{A. SCMF analysis with simulated structures}

The effective thermal conductivity is plotted as a function of shear strain in Fig. 1 for simulations with $\alpha=100$ and several different concentrations, for a Mason number of $3.125 \times 10^{-2}$. In each run, the equations of motion were first 


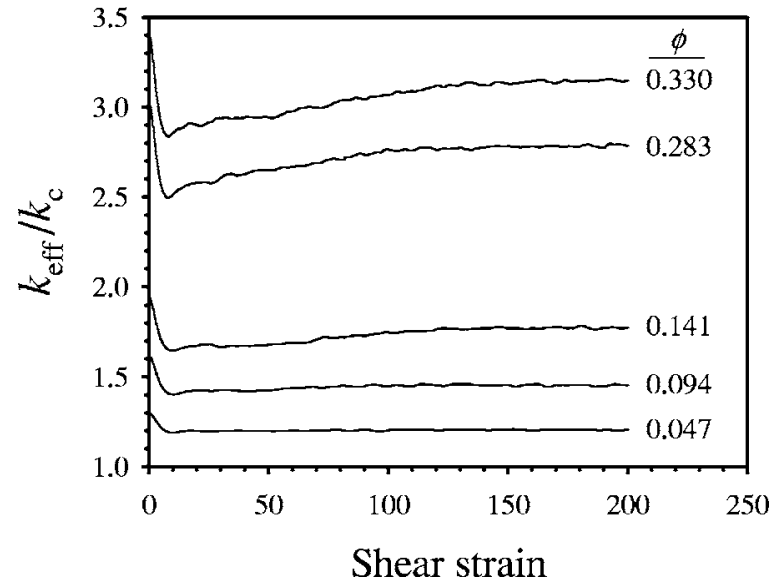

FIG. 1. Effective conductivity as a function of shear strain for several different particle volume fractions at a Mason number of $\mathrm{Mn}=3.125 \times 10^{-2}$, using the self-consistent point-dipole model $(\alpha=100)$.

integrated numerically with $\mathrm{Mn}=0$ to obtain static, anisotropic structures. Shear flow was then simulated, and the effective thermal conductivity was calculated every strain interval of 0.1. For all concentrations at this value of $\mathrm{Mn}$, $k_{\text {eff }} / k_{c}$ initially decreases with increasing shear strain, as the chain-like structures formed initially are degraded to some extent by the shear flow. For all but the smallest concentrations, $k_{\text {eff }} / k_{c}$ then increases with increasing strain. This occurs as the particles form lamellar aggregates, or stripes, oriented in the flow direction. Such structures have been observed previously in experiments, ${ }^{48-53}$ as well as particle-level $^{5,55}$ and continuum ${ }^{53,56,57}$ models of ER and MR suspensions. The impact of these lamellar structure will be discussed further below. In all cases, $k_{\mathrm{eff}} / k_{c}$ eventually reaches a steady value. After reaching steady state, results are averaged over a strain of 10 to obtain values for the steady-state effective conductivities. The steady values are discussed below.

The steady-state effective thermal conductivities obtained from the simulations with the SCMF model are plotted as a function of Mason number for different volume fractions and $\alpha=100$ in Fig. 2. At each concentration, $k_{\text {eff }} / k_{c}$ increases as $\mathrm{Mn}$ is decreased. These results agree qualitatively with the experimental results reported for the shear rate and field strength dependence of the dielectric permittivity and electrical conductivity of ER suspensions. ${ }^{17,25-28}$ At small Mn, hydrodynamic forces are relatively weak, and thus columnar structures that are broken by the straining motion can rapidly reform. On average, the structure is highly anisotropic which results in a large effective thermal conductivity. For large Mn, hydrodynamic forces are much stronger, and the columnar structures are more degraded. The suspension structure is thus more isotropic and possesses a smaller effective thermal conductivity. At a specific value of $\mathrm{Mn}$, the effective thermal conductivity increases with $\phi$, as expected for suspensions of highly conducting particles.

The data in Fig. 2 are well represented by the empirical expression

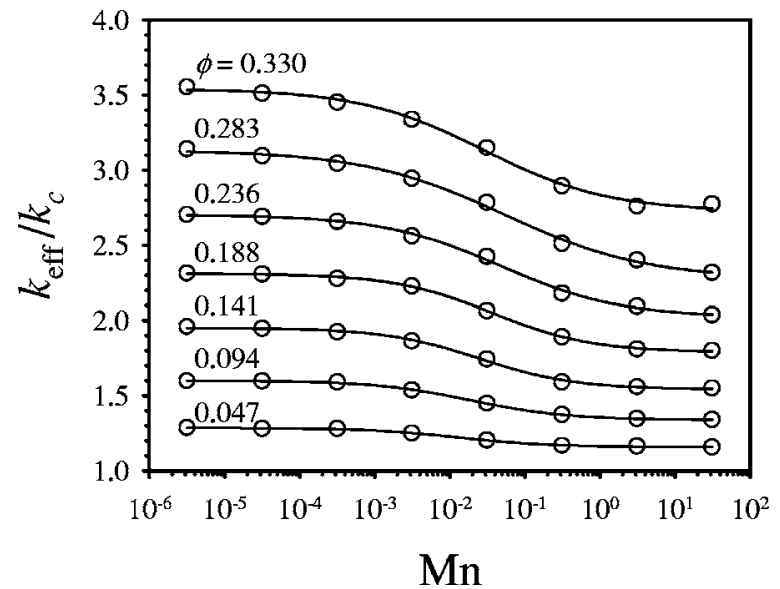

FIG. 2. Effective conductivity as a function of Mason number for several different particle volume fractions, using the self-consistent point-dipole model $(\alpha=100)$. The solid curves represent least-squares fits of Eq. (27) to the simulation data.

$$
k_{\mathrm{eff}}(\mathrm{Mn}, \phi, \alpha)=\frac{k_{0}(\phi, \alpha)-k_{\infty}(\phi, \alpha)}{1+(\lambda \mathrm{Mn})^{n}}+k_{\infty}(\phi, \alpha),
$$

where $k_{0}(\phi, \alpha)$ and $k_{\infty}(\phi, \alpha)$ are the values of $k_{\text {eff }}$ in the limits of small and large Mn, respectively, and $\lambda$ and $n$ are parameters that depend weakly on $\phi$ and $\alpha$. Best-fit values of these parameters for $\alpha=100$ are tabulated as a function of $\phi$ in Table II. The solid curves in Fig. 2 are from Eq. (27) using the parameter values in Table II. Below we obtain corrections for the values of $k_{\text {eff }}$ obtained using the SCMF model to account for effects of higher order thermal multipoles on energy transport. At that point, we will present values of $k_{0}$ and $k_{\infty}$ for other values of $\alpha$.

In Fig. $3, k_{\text {eff }} / k_{c}$ for simulations at $\phi=0.094$ and $\alpha$ $=100$ is plotted as a function of Mn. Also shown are the predictions for the perfect chain structure using the SCMF model, as well as the Maxwell model [Eq. (1)], and snapshots of the suspension at different Mn. At small Mn, $k_{\text {eff }} / k_{c}$ from the simulations is similar to that predicted by the perfect chain model. This is consistent with the observation that the structure is indeed anisotropic with chain-like structures apparent at $\mathrm{Mn}=3.125 \times 10^{-6}$. At large $\mathrm{Mn}, k_{\mathrm{eff}} / k_{c}$ approaches that predicted by the Maxwell model. This is not unexpected since the Maxwell model is an approximate solution to the SCMF point-dipole model assuming $g(r)=1$ for

TABLE II. Parameters $k_{0}, k_{\infty}, \lambda$ and $n$ as a function of particle volume fraction (for $\alpha=100$ ).

\begin{tabular}{ccccc}
\hline \hline$\phi$ & $k_{0} / k_{c}$ & $k_{\infty} / k_{c}$ & $\lambda$ & $n$ \\
\hline 0.047 & 1.29 & 1.16 & 77.5 & 0.715 \\
0.094 & 1.60 & 1.34 & 53.1 & 0.671 \\
0.141 & 1.95 & 1.54 & 39.9 & 0.682 \\
0.188 & 2.31 & 1.79 & 26.9 & 0.656 \\
0.236 & 2.70 & 2.02 & 20.7 & 0.544 \\
0.283 & 3.13 & 2.27 & 18.6 & 0.459 \\
0.330 & 3.54 & 2.73 & 35.3 & 0.523 \\
\hline \hline
\end{tabular}




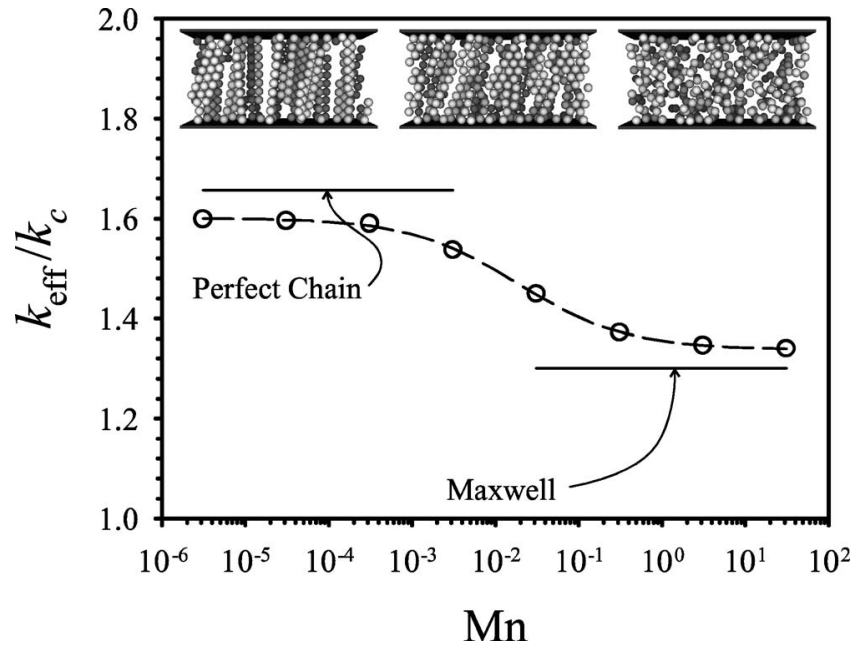

FIG. 3. Effective conductivity as a function of Mason number for $\phi$ $=0.094(\alpha=100)$. Also shown are the values of $k_{\text {eff }} / k_{c}$ predicted using the perfect chain and Maxwell models, and snapshots of the suspension at three different Mason numbers $\left(\mathrm{Mn}=3.125 \times 10^{-6}, 3.125 \times 10^{-3}\right.$ and 31.25 , from left to right).

$r>\sigma$. The difference between the Maxwell model and the large $\mathrm{Mn}$ results are likely attributed to the fact that $g(r)$ $\neq 1$ in the large Mn simulations.

In Fig. $4, k_{\text {eff }} / k_{c}$ for simulations at $\phi=0.330$ and $\alpha$ $=100$ is plotted as a function of Mn. Again, also shown are the predictions for the perfect chain structure using the SCMF model, as well as the Maxwell model. In this case, the simulation results are significantly larger than the values predicted by the SCMF perfect chain and Maxwell models in the respective limits. The discrepancy can be explained by examining the suspension structure under these conditions. A snapshot of the simulated structure obtained for $\phi=0.330$ after shearing with $\mathrm{Mn}=9.375 \times 10^{-2}$ to a strain of 200 is shown in Fig. 5. In this snapshot, the flow direction is into the page; the microstructure has organized into lamellar aggregates, or stripes, oriented in the flow direction. While the structure is still anisotropic, isolated particle chains no longer exist, but rather are interconnected into much larger struc-

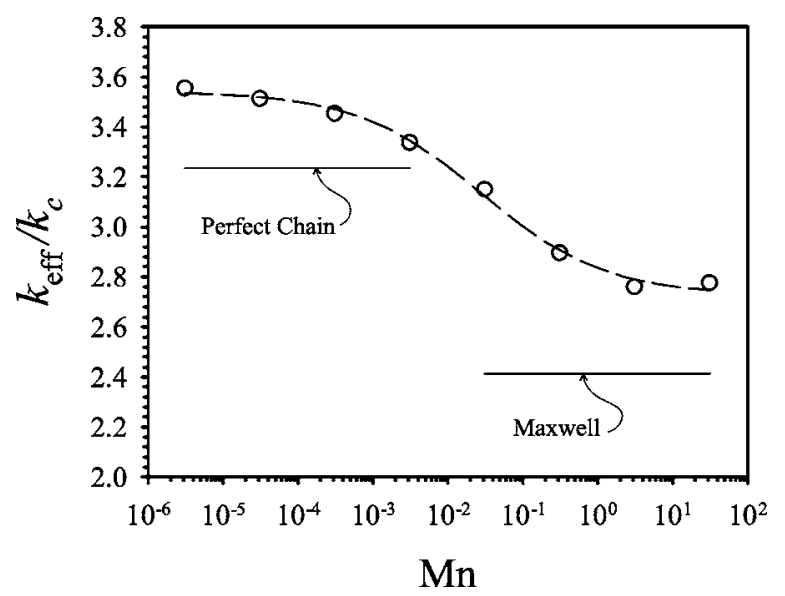

FIG. 4. Effective conductivity as a function of Mason number for $\phi$ $=0.330(\alpha=100)$. Also shown are the values of $k_{\text {eff }} / k_{c}$ predicted by the perfect chain and Maxwell models.

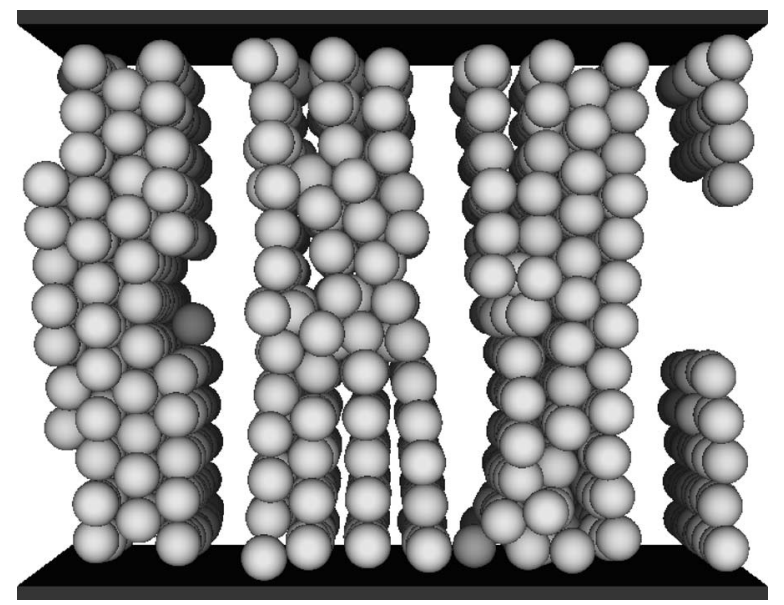

FIG. 5. Snapshot of the suspension microstructure for $\phi=0.330$ after shearing to a strain of 200 with $\mathrm{Mn}=9.375 \times 10^{-2}$. The flow direction is into the page. For this simulation, $L_{x} \times L_{y} \times L_{z}=10 \sigma \times 15 \sigma \times 13 \sigma$.

tures. The effective thermal conductivities are larger than expected for such structures because in the neighborhood of a particle, the local volume fraction is larger than the average. Since models typically predict effective thermal conductivities with $\partial^{2} k_{\mathrm{eff}} / \partial \phi^{2}>0$ [see, for example, Eqs. (1)-(3)], we expect the heterogeneous suspensions with lamellar structures to possess larger effective thermal conductivities than homogeneous structures under otherwise similar conditions. These observations also explain why a slow transient increase in $k_{\text {eff }}$ is observed in the simulations; $k_{\text {eff }}$ increases slowly as the lamellar structures form. These results suggest that measuring effective thermal conductivities, or analogous electric or magnetic properties, under shear may be a useful method for probing the lamellar structure formation process.

\section{B. Corrections for multipole effects}

The SCMF point-dipole approximation for the thermal dipole strength underestimates the effective thermal conductivity of a suspension. This is illustrated in Fig. 6, where the effective thermal conductivity for $\phi=0.330$ and $\alpha=100$ is

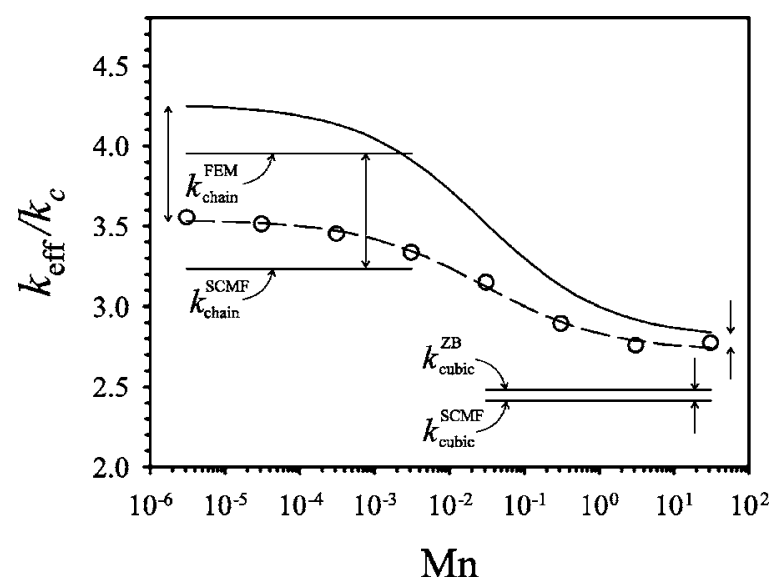

FIG. 6. Effective conductivity as a function of Mason number for $\phi$ $=0.330(\alpha=100)$. The symbols represent the simulation data, the dashed curve represents the fit with Eq. (27), and the solid curve represents Eq. (27) using the corrected values $k_{0}^{\text {corr }}$ and $k_{\infty}^{\text {corr }}$. 
presented for the SCMF model for simulated suspension structures, as well as for the perfect chain and cubic lattice structures using both the SCMF model and more accurate solutions for the temperature distribution and effective thermal conductivity. For the perfect chain structure, the finiteelement solution produces an effective thermal conductivity $\left(k_{\text {chain }}^{\mathrm{FEM}}\right)$ that is significantly larger than that obtained using the SCMF point-dipole solution $\left(k_{\text {chain }}^{\mathrm{SCMF}}\right)$. The difference is attributed to higher-order multipole contributions to the temperature distribution that are captured by the FEM solution but not the SCMF point-dipole solution. The effect is significant, resulting in an increase in $k_{\text {chain }}$ of $22 \%$. Also illustrated in Fig. 6 is a comparison of the SCMF point-dipole model with a more accurate solution for cubic structures $\left(k_{\text {cubic }}^{\mathrm{SCMF}}\right.$ and $k_{\text {cubic }}^{\mathrm{ZB}}$ ). Here, we employ Eq. (3) for the more accurate solution; this expression agrees with finite-element solutions within $2 \%$ for the ranges of parameters considered here. Figure 6 illustrates that for the cubic structure, the influence of higher-order thermal multipoles is less significant than for the chain structures, resulting in only a $2.9 \%$ increase in $k_{\text {cubic }}$ (for this case, $k_{\text {cubic }}^{\mathrm{ZB}}$ and $k_{\text {cubic }}^{\mathrm{FEM}}$ differ by less than $0.1 \%$ ). This is not surprising, since the effects of higher order multipoles are known to be most important when particle pair separations are small. ${ }^{7,34,35}$

In principle, one could solve more accurately for the temperature within the simulated structures to obtain values for $k_{\text {eff }}$ that include contributions from the higher-order multipoles. This task, however, would be very computationally demanding and is outside of the scope of this work. We can, however, approximately account for the effects of higherorder multipoles as follows. Consider the case of sheared suspensions at small $\mathrm{Mn}$. The suspension structure in this limit is similar to that of the perfect chain structure, with columnar structures extending from one surface to the other (see Fig. 3). For the perfect chain structure, the effective thermal conductivity obtained using the SCMF model can be "corrected" exactly by simply adding the difference $k_{\text {chain }}^{\mathrm{FEM}}$ $-k_{\mathrm{chain}}^{\mathrm{SCMF}}$ (for each value of $\phi$ and $\alpha$ ). We therefore correct the values obtained for $k_{\text {eff }}$ from simulations in the limit of small Mn with the SCMF point-dipole model by adding the same difference. This corresponds to correcting the parameter $k_{0}$ in Eq. (27) fit to simulation data using

$$
k_{0}^{\text {corr }}(\phi, \alpha)=k_{0}(\phi, \alpha)+\left[k_{\text {chain }}^{\mathrm{FEM}}(\phi, \alpha)-k_{\text {chain }}^{\mathrm{SCMF}}(\phi, \alpha)\right] .
$$

At large $\mathrm{Mn}$, the chain-like aggregates are destroyed, resulting in a suspension structure for which nearestneighbor particle pairs are separated by larger distances on average. In order to correct the values of $k_{\text {eff }}$ obtained from simulations to account for higher-order multipoles, we model the large Mn microstructure as a cubic lattice. The values of $k_{\text {eff }}$ obtained from the simulations can thus be corrected by adding the difference $k_{\text {cubic }}^{\mathrm{ZB}}-k_{\text {cubic }}^{\mathrm{SCMF}}$ (for each value $\phi$ and $\alpha$ ). This corresponds to correcting the parameter $k_{\infty}$ in Eq. (27) fit to simulation data using

$$
k_{\infty}^{\text {corr }}(\phi, \alpha)=k_{\infty}(\phi, \alpha)+\left[k_{\text {cubic }}^{\mathrm{ZB}}(\phi, \alpha)-k_{\text {cubic }}^{\mathrm{SCMF}}(\phi, \alpha)\right] .
$$

The values of $k_{0}^{\text {corr }}$ and $k_{\infty}^{\text {corr }}$ obtained as described above are plotted as a function of $\phi$ for various values of $\alpha$ in Fig. 7. The values of $n$ and $\lambda$ obtained from fitting Eq. (27) to the

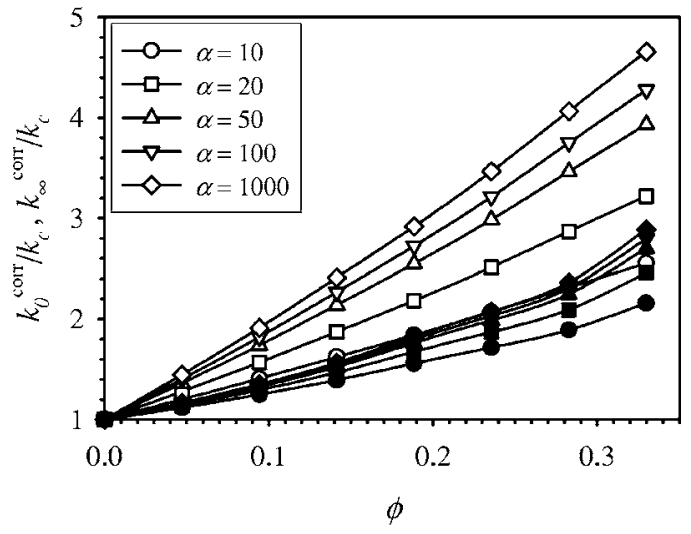

FIG. 7. $k_{0}^{\text {corr }}$ (open symbols) and $k_{\infty}^{\text {corr }}$ (filled symbols) as a function of $\phi$ for different values of $\alpha$.

simulation data are plotted in Fig. 8. These parameters can be used to predict via Eq. (27) values for $k_{\text {eff }}$ as a function of $\mathrm{Mn}, \phi$, and $\alpha$. The behavior predicted for $k_{\mathrm{eff}}(\mathrm{Mn})$ for $\phi$ $=0.330$ and $\alpha=100$ using the corrected parameters is illustrated in Fig. 6. The values of $k_{0}^{\text {corr }}$ and $k_{\infty}^{\text {corr }}$ in Fig. 7 predict that for large $\alpha$, the effective thermal conductivity of a suspension should roughly double as $\mathrm{Mn}$ is decreased from the large to the small $\mathrm{Mn}$ regimes.

\section{CONCLUSION}

We have analyzed thermal transport in sheared ER and MR fluids. Although viscous dissipation can be significant, energy transport on the particle length scale can be analyzed by ignoring energy production by viscous dissipation. For typical situations, energy transport normal to the flow direction is dominated by conduction.

Particle-level simulations were employed to determine the suspension structure as a function of $\mathrm{Mn}$ and $\phi$. A selfconsistent mean-field (SCMF) dipole model is used to estimate the $z z$ component effective thermal conductivities, $k_{\text {eff }}$, for these simulated structures (where the external field is applied in the $z$ direction). The field-induced chain-like aggregates that form at small $\mathrm{Mn}$ result in a larger effective thermal conductivity at small $\mathrm{Mn}$ than at large $\mathrm{Mn}$. The dependence of $k_{\text {eff }}$ on Mn, volume fraction $\phi$, and conductivity

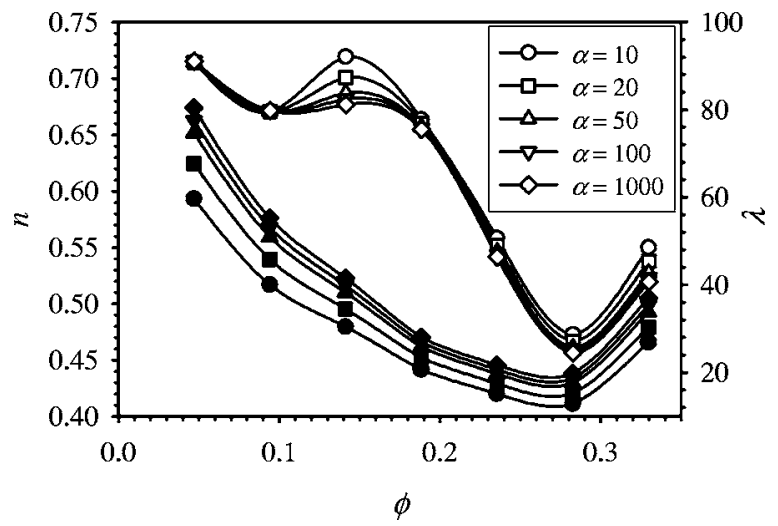

FIG. 8. $n$ (open symbols) and $\lambda$ (filled symbols) as a function of $\phi$ for different values of $\alpha$. 


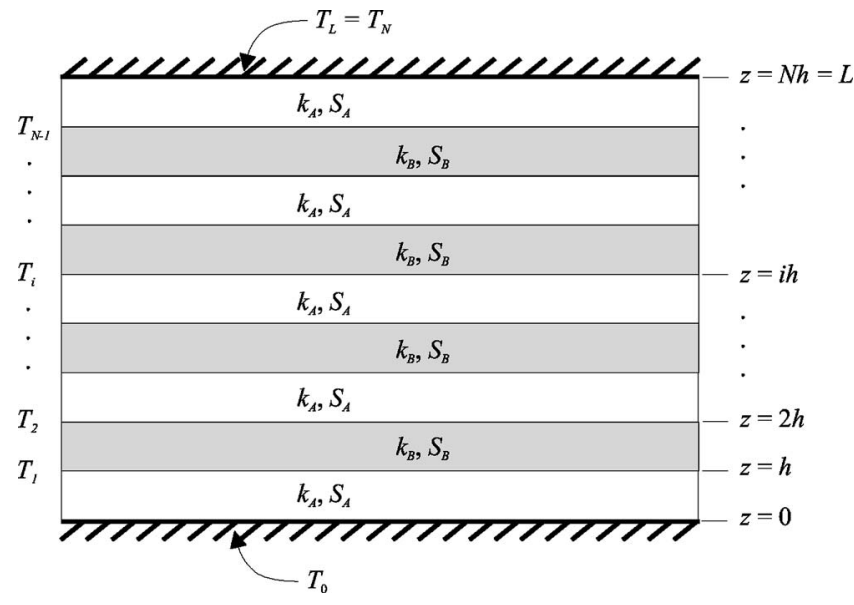

FIG. 9. Schematic diagram of a one-dimensional composite composed of alternating layers of materials $A$ and $B$.

ratio $\alpha$ is well represented by the empirical form $k_{\mathrm{eff}}(\mathrm{Mn}, \phi, \alpha)=\left(k_{0}(\phi, \alpha)-k_{\infty}(\phi, \alpha)\right) /\left(1+(\lambda \mathrm{Mn})^{n}\right)+k_{\infty}(\phi, \alpha)$. This form qualitatively reproduces prior experimental results for the electrical properties of sheared ER suspensions.

In the SCMF thermal analysis, the particles were assumed to only generate dipole disturbances to the temperature profile. Effects of higher-order multipoles were estimated by comparing effective thermal conductivities of model structures obtained using the SCMF and more accurate methods (e.g., the finite-element method). For large $\alpha$, the effective thermal conductivity of a sheared ER or MR suspension is predicted to roughly double as $\mathrm{Mn}$ is decreased from the large to the small Mn limits.

\section{APPENDIX: CONDUCTION AND ENERGY PRODUCTION IN A ONE-DIMENSIONAL COMPOSITE}

In this appendix we show that for the case of a onedimensional composite, the effective conductivity calculated obtained in the absence of energy production can be used to describe the temperature profile and energy flux when energy production is present.

Consider a one-dimensional composite composed of alternating layers of materials $A$ and $B$ as depicted in Fig. 9. The materials $A$ and $B$ have thermal conductivities $k_{A}$ and $k_{B}$, and energy production rates per unit volume $S_{A}$ and $S_{B}$, respectively. Each layer has the same thickness $h$.

Consider first the temperature profile obtained by solution of energy equation within each layer $i$,

$$
0=k_{i} \frac{d^{2} T^{(i)}}{d z^{2}}+S_{i},
$$

subject to the boundary conditions of continuity of temperature and conductive flux at each interface $z_{i}$. The solution can be expressed in terms of the interfacial temperatures $T_{i}$ $=T^{(i)}\left(z_{i}\right)$,
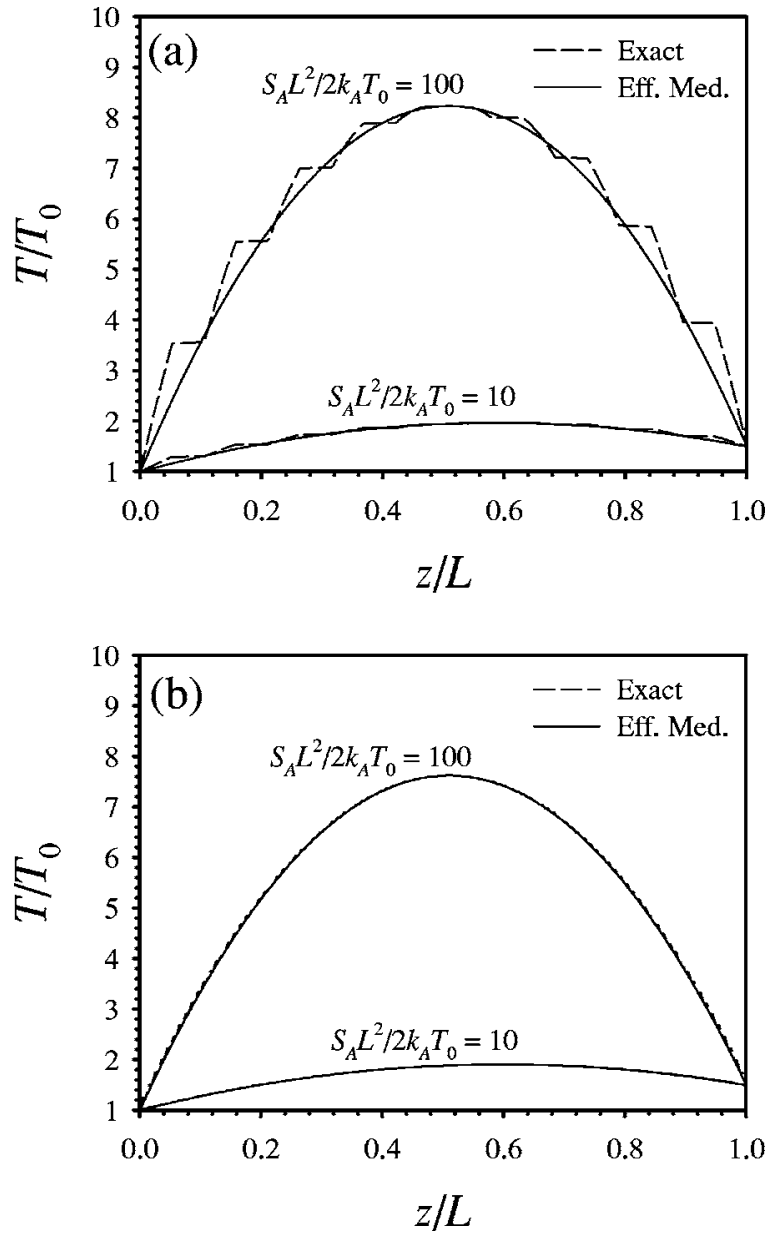

FIG. 10. Temperature profiles $T / T_{0}$ vs $z / L$ for two different values of $S_{A} L^{2} / 2 k_{A} T_{0}$ (with $T_{L} / T_{0}=1.5, S_{B}=0$, and $k_{B} / k_{A}=100$ ) for the exact and effective medium solutions [Eqs. (A2) and (A14), respectively]. (a) $N=L / h$ =19; (b) $N=L / h=199$.

$$
\begin{aligned}
T^{(i)}(z)= & -\frac{S_{i} h^{2}}{2 k_{i}}\left(\frac{z}{h}\right)^{2}+\left[T_{i}-T_{i-1}+\frac{S_{i} h^{2}}{2 k_{i}}(2 i-1)\right] \frac{z}{h} \\
& +\left[i T_{i-1}-(i-1) T_{i}-\frac{S_{i} h^{2}}{2 k_{i}}\left(i^{2}-i\right)\right],
\end{aligned}
$$

where the interfacial temperatures are given by the solution of the system of equations

$$
-k_{i} T_{i-1}+\left(k_{i}+k_{i+1}\right) T_{i}-k_{i+1} T_{i+1}=\frac{h^{2}}{2}\left(S_{i}+S_{i+1}\right),
$$

for $i=1$ to $N-1$, along with given boundary temperatures $T_{0}$ and $T_{N}=T_{L}$. Example temperature profiles are illustrated in Fig. 10.

Next consider the problem of determining the effective composite conductivity when the energy production terms are zero $\left(S_{i}=0\right.$ for all $\left.i\right)$. At steady state, the conductive energy flux is constant throughout. The differences between interface temperatures across each layer can thus be written

$$
T_{1}-T_{0}=-q_{z} \frac{h}{k_{A}},
$$




$$
\begin{aligned}
& T_{2}-T_{1}=-q_{z} \frac{h}{k_{B}}, \\
& T_{3}-T_{2}=-q_{z} \frac{h}{k_{A}}, \\
& \vdots \\
& T_{N}-T_{N-1}=-q_{z} \frac{h}{k_{B}} .
\end{aligned}
$$

Adding these equations gives the energy flux in terms of the boundary temperatures

$$
q_{z}=-k_{\mathrm{eff}} \frac{T_{N}-T_{0}}{L}
$$

where the effective medium thermal conductivity is

$$
k_{\mathrm{eff}}=k_{a}\left[\frac{1}{2}\left(\frac{k_{A}}{k_{B}}+1\right)+\frac{1}{2 N}\left(1-\frac{k_{A}}{k_{B}}\right)\right]^{-1} .
$$

Finally, consider the effective medium temperature profile $\langle T\rangle$, governed by the energy balance

$$
0=k_{\mathrm{eff}} \frac{d^{2}\langle T\rangle}{d z^{2}}+\langle S\rangle,
$$

where

$$
\begin{aligned}
\langle S\rangle & =\frac{1}{L} \int_{0}^{L} S \mathrm{~d} z \\
& =\frac{1}{2}\left(S_{A}+S_{B}\right)+\frac{1}{2 N}\left(S_{A}-S_{B}\right)
\end{aligned}
$$

is the effective medium energy production rate. Using the boundary conditions $\langle T\rangle=T_{0}$ at $z=0$ and $\langle T\rangle=T_{L}$ at $z=L$, the resulting temperature profile is

$$
\langle T\rangle(z)=\frac{\langle S\rangle L^{2}}{2 k_{\text {eff }}}\left[\frac{z}{L}-\left(\frac{z}{L}\right)^{2}\right]+\left(T_{L}-T_{0}\right) \frac{z}{L}+T_{0} .
$$

Temperature profiles obtained from the exact and effective medium solutions [Eqs. (A2) and (A14), respectively] are plotted in Fig. 10 for two different values of $N(N=19$ and 199), using the parameter values $k_{B} / k_{A}=100$, $S_{A} L^{2} / 2 k_{A} T_{0}=100, S_{B}=0$, and $T_{L} / T_{0}=1.5$. As $N$ increases ( $h / L$ decreases), the effective medium profile approaches that obtained from the exact solution. Thus, as with the case for a system without energy production, the effective medium approximation only provides a reasonable approximation to the temperature profile when $h / L \ll 1$.

We note also that even for $S_{A} L^{2} / 2 k_{A} T_{0}=100$, the dimensionless energy production term appearing in Eq. (7) is smaller by a factor of $\mathcal{O}\left[(h / L)^{2}\right]$. Thus, even though the $S_{A}$ may be large enough to significantly increase the temperature distribution within the composite, the profiles on the length scale of the heterogeneities are essentially linear (for small $h / L)$. Thus, in this limit $(h / L \rightarrow 0)$, the effective medium conductivity obtained in the absence of energy production provides a good estimate of the energy flux and temperature profile even when energy production is present.

${ }^{1}$ D. L. Hartsock, R. F. Novak, and G. J. Chaundy, "ER fluid requirements for automotive devices," J. Rheol. 35, 1305 (1991).

${ }^{2}$ J. M. Ginder, "Rheology controlled by magnetic fields," in Encyclopedia of Applied Physics, edited by G. L. Trigg (VCH, Weinheim, 1996), Vol. 16, p. 487.

${ }^{3}$ J. D. Carlson and J. L. Sproston, "Controllable fluids in 2000-status of ER and MR fluid technology," in Actuator 2000-7th International Conference on New Actuators, edited by H. Borgmann (Messe Bremen GmbH, Bremen, 2000), pp. 126-130.

${ }^{4}$ D. J. Klingenberg, "Magnetorheology: Applications and challenges," AIChE J. 47, 246 (2001).

${ }^{5}$ J. M. Ginder, L. C. Davis, and L. D. Elie, "Rheology of magnetorheological fluids: Models and measurement," Int. J. Mod. Phys. B 23, 3293 (1996).

${ }^{6}$ M. R. Jolly, J. W. Bender, and J. D. Carlson, "Properties and applications of commercial magnetorheological fluids," J. Intell. Mater. Syst. Struct. 10, 5 (1999).

${ }^{7}$ D. J. Jeffrey, "Conduction through a random suspensions of spheres," Proc. R. Soc. London, Ser. A 335, 355 (1973).

${ }^{8}$ Y. C. Chiew and E. D. Glandt, "The effect of structure on the conductivity of a dispersion," J. Colloid Interface Sci. 94, 90 (1983).

${ }^{9}$ J. C. R. Turner, "Two-phase conductivity. The electrical conductance of liquid-fluidized beds of spheres," Chem. Eng. Sci. 31, 487 (1976).

${ }^{10} \mathrm{M}$. Zuzovsky and H. Brenner, "Effective conductivities of composite materials composed of cubic arrangements of spherical particles embedded in an isotropic matrix," J. Appl. Math. 28, 979 (1977).

${ }^{11}$ R. F. Meredith and C. W. Tobias, "Resistance to potential flow through a cubical array of spheres," J. Appl. Phys. 31, 1270 (1960).

${ }^{12}$ A. S. Ahuja, "Augmentation of heat transport in laminar flow of polystyrene suspensions. I. Experiments and results," J. Appl. Phys. 46, 3408 (1975).

${ }^{13}$ A. S. Ahuja, "Augmentation of heat transport in laminar flow of polystyrene suspensions. II. Analysis of the data," J. Appl. Phys. 46, 3417 (1975).

${ }^{14}$ S. Shin and S. H. Lee, "Thermal conductivity of suspensions in shear flow fields," Int. J. Heat Mass Transfer 43, 4275 (2000).

${ }^{15}$ A. L. Zydney and C. K. Colton, "Augmented solute transport in the shear flow of a concentrated suspension," PCH, PhysicoChem. Hydrodyn. 10, 77 (1988).

${ }^{16}$ H. E. Hollmann, "Semiconductive colloidal suspensions with nonlinear properties," J. Appl. Phys. 21, 402 (1950).

${ }^{17}$ T. Y. Chen and P. F. Luckham, "A study of the electrical current passing through water-activated electro-rheological fluids," J. Phys. D 27, 1556 (1994).

${ }^{18} \mathrm{~W}$. Wen, S. Men, and K. Lu, "Structure-induced nonlinear dielectric properties in electrorheological fluids," Phys. Rev. E 55, 3015 (1997).

${ }^{19} \mathrm{D}$. Adolph and T. Garino, "Time-dependent dielectric response of quiescent electrorheological fluids," Langmuir 11, 307 (1995).

${ }^{20}$ J. M. Ginder, M. E. Nichols, L. D. Elie, and J. L. Tardiff, "Magnetorheological elastomers: Properties and applications," Proc. SPIE 3675, 131 (1999).

${ }^{21}$ J. de Vicente, G. Bossis, S. Lacis, and M. Guyot, "Permeability measurements in cobalt ferrite and carbonyl iron powders and suspensions," J. Magn. Magn. Mater. 251, 100 (2002).

${ }^{22}$ L. C. Davis, "Ground state of an electrorheological fluid," Phys. Rev. A 46, R719 (1992).

${ }^{23}$ T. B. Jones, Electromechanics of Particles (Cambridge University Press, Cambridge, 1995).

${ }^{24}$ Y. M. Shkel and D. J. Klingenberg, "Magnetorheology and magnetostriction of isolated chains of nonlinear magnetizable spheres," J. Rheol. 45, 351 (2001).

${ }^{25}$ E. Lemaire, G. Bossis, and Y. Grasselli, "Rheological behavior of electrorheological fluids," Langmuir 8, 2957 (1992).

${ }^{26}$ D. Adolph, T. Garino, and B. Hance, "Permittivity of electrorheological fluids under steady and oscillatory shear," Langmuir 11, 313 (1995).

${ }^{27}$ O. Quadrat and J. Stejskal, "Electrical conductivity of flowing polyaniline suspensions," J. Rheol. 42, 13 (1998).

${ }^{28}$ Y. Saimoto, T. Satoh, and M. Konno, "Correlation between generated shear stress and generated permittivity for the electrorheological response 
of colloidal silica suspensions," J. Colloid Interface Sci. 219, 135 (1999).

${ }^{29}$ R. B. Bird, W. E. Stewart, and E. N. Lightfoot, Transport Phenomena, 2nd ed. (Wiley, New York, 2002).

${ }^{30}$ F. P. Incropera and D. P. De Witt, Fundamentals of Heat and Mass Transfer, 3rd ed. (Wiley, New York, 1990).

${ }^{31}$ J. P. Holman, Heat Transfer, 9th ed. (McGraw-Hill, New York, 2002).

${ }^{32}$ CRC Handbook of Chemistry and Physics, edited by R. C. Weast (CRC, Boca Raton, 1981).

${ }^{33}$ G. K. Batchelor, "Transport properties of two-phase materials with random structure," Annu. Rev. Fluid Mech. 6, 227 (1974).

${ }^{34}$ G. K. Batchelor and R. W. O'Brien, "Thermal or electrical conduction through a granular material," Proc. R. Soc. London, Ser. A 355, 313 (1977).

${ }^{35}$ R. T. Bonnecaze and J. F. Brady, "A method for determining the effective conductivity of dispersions of particles," Proc. R. Soc. London, Ser. A 430, 285 (1990)

${ }^{36}$ D. J. Klingenberg, F. van Swol, and C. F. Zukoski, "The small shear rate response of electrorheological suspensions. I. Simulation in the pointdipole limit," J. Chem. Phys. 94, 6160 (1991).

${ }^{37}$ D. J. Klingenberg, "Simulation of the dynamic response of electrorheological suspensions: Demonstration of a relaxation mechanism," J. Rheol. 37, 199 (1993).

${ }^{38}$ M. Parthasarathy and D. J. Klingenberg, "A microstructural investigation of the nonlinear response of electrorheological suspensions I. Start-up of steady shear flow," Rheol. Acta 34, 417 (1995).

${ }^{39}$ M. Parthasarathy and D. J. Klingenberg, "Electrorheology: Mechanisms and models," Mater. Sci. Eng., R. 17, 57 (1996).

${ }^{40}$ Y. M. Shkel and D. J. Klingenberg, "Magnetorheology and magnetostriction of isolated chains of ferromagnetic spheres," J. Rheol. 45, 351 (2001).

${ }^{41}$ D. Kittipoomwong, D. J. Klingenberg, and J. C. Ulicny, "Simulation of bidisperse magnetorheological fluids," Int. J. Mod. Phys. B 16, 2732 (2002).

${ }^{42}$ D. J. Klingenberg, F. van Swol, and C. F. Zukoski, "The small shear rate response of electrorheological suspensions. II. Extension beyond the point-dipole limit," J. Chem. Phys. 94, 6170 (1991).
${ }^{43}$ R. T. Bonnecaze and J. F. Brady, "Dynamic simulation of an electrorheological fluid," J. Chem. Phys. 96, 2183 (1992).

${ }^{44}$ Y. Baxter-Drayton and J. F. Brady, "Brownian electrorheological fluids as a model for flocculated dispersions," J. Rheol. 40, 1027 (1996).

${ }^{45}$ P. M. Adriani and A. P. Gast, "A microscopic model of electrorheology," Phys. Fluids 31, 2757 (1988).

${ }^{46}$ J. M. Ginder, "Behavior of magnetorheological fluids," MRS Bull. 23, 26 (1998).

${ }^{47}$ M. P. Allen and D. J. Tildesley, Computer Simulation of Liquids (Oxford University Press, Oxford, 1987).

${ }^{48}$ G. Bossis, E. Lemaire, J. Persello, and L. Petit, "Structuration and elasticity of electrorheological fluids," Prog. Colloid Polym. Sci. 89, 1 (1992).

${ }^{49}$ G. Bossis, Y. Grasselli, E. Lemaire, J. Persello, and L. Petit, "Phase separation and flow-induced anisotropy in electrorheological fluids," Europhys. Lett. 25, 335 (1994).

${ }^{50} \mathrm{~S}$. Henley and F. E. Filisko, "Flow profiles of electrorheological suspensions: An alternative model for ER activity," J. Rheol. 43, 1323 (1999).

${ }^{51}$ O. Volkova, S. Cutillas, and G. Bossis, "Shear banded flows and nematicto-isotropic transition in ER and MR fluids," Phys. Rev. Lett. 82, 233 (1999).

${ }^{52}$ S. Vieira, L. B. Pompeo Neto, and A. C. F. Arruda, "Transient behavior of an electrorheological fluid in shear flow mode," J. Rheol. 44, 1139 (2000).

${ }^{53}$ K. von Pfeil and D. J. Klingenberg, "Nonlocal electrostatics in heterogeneous suspensions using a point-dipole model," J. Appl. Phys. 96, 5341 (2004).

${ }^{54}$ J. R. Melrose and D. M. Heyes, "Simulations of electrorheological and particle mixture suspensions: Agglomerate and layer structures," J. Chem. Phys. 98, 5873 (1993).

${ }^{55}$ J. E. Martin, "Thermal chain model of electrorheology and magnetorheology," Phys. Rev. E 63, 011406 (2001).

${ }^{56}$ K. von Pfeil, M. D. Graham, D. J. Klingenberg, and J. F. Morris, "Pattern formation in flowing electrorheological fluids," Phys. Rev. Lett. 88, 188301 (2002)

${ }^{57}$ K. von Pfeil, M. D. Graham, D. J. Klingenberg, and J. F. Morris, "Structure evolution in electrorheological and magnetorheological suspensions from a continuum perspective," J. Appl. Phys. 93, 5769 (2003). 